\title{
Three-dimensional cellular automaton model for the destruction of brittle heterogeneous materials
}

\author{
Galina Kazunina ${ }^{1 *}$ and Allay Cherednichenko ${ }^{1}$
}

${ }^{1}$ T.F. Gorbachev Kuzbass State Technical University, Kemerovo, Russia.

\begin{abstract}
The article investigates the evolution modes of cluster damage structure in brittle heterogeneous materials by using a three-dimensional probabilistic cellular automaton. By comparing the data of computer and physical experiments, there was established the essential role of the model parameter, which describes the intensity of the material destruction process under the influence of local overstress near the existing damage clusters - the probability of perimeter germination. The comparison of kinetic curves of damage accumulation and correlation functions showed that, depending on the probability value for damage cluster perimeter germination, two qualitatively different modes of evolution of damage accumulation process are observed. In this case, the best correspondence of correlation functions in model and physical experiment on pulsed electromagnetic emission is observed for perimeter germination probability values smaller than 0.2. Key words: cellular automaton, prognostication of destruction, pulsed emission.
\end{abstract}

\section{Introduction}

Modern development of methods for monitoring the mode of deformation and predicting the destruction of rocks basing on pulsed electromagnetic emission [1] requires not only the improvement of experimental techniques, but also the development of new approaches to interpreting experimental data. This is due to the fact that the observed process of pulsed emission registers the release of energy during the formation of each new fracture fixing the formation of new ones or "germination" of already existing areas and carries information about the kinetic process of damage accumulation. But the characteristics of pulsed emission give only indirect information about the spatial distribution of damage and the dynamics of its cluster structure, which is of primary interest for predicting destruction. Simultaneous observation of damage accumulation and cluster structure formed by it in dynamics is not possible at the current level of technology development. Such a study can be carried out by computer simulation methods. The existence of general laws at the stage preceding the destruction of the material also testifies in favour of this approach [2].

\footnotetext{
${ }^{1}$ * Corresponding author: gt-kga@yandex.ru
} 
According to the acoustic experiment, microcracks, for example, in rocks are formed mainly at an intermediate or mesoscopic level, and, therefore, the process of transition of destruction to a macroscopic level can be described in essence without referring to the details of the dynamics of individual elementary acts, but relying only on the geometric characteristics of the structure under consideration (percolation models). In [3, 4], a cellular automaton model was constructed on the basis of the kinetic theory of strength which considers the kinetics of damage accumulation as a unified spatial and temporal process taking into account the internal dynamics. The model allows studying separately, but synchronously and consistently, the kinetics of newly occurring fractures (analogues of emission pulses in a physical experiment) and the spatial and temporal dynamics of the damage clusters configuration formed by them, obtaining statistical characteristics of processes such as correlation functions, Hurst statistics, cluster size distribution functions. The presence of simultaneously simulated characteristics of the destruction process makes it possible to reasonably compare the statistical characteristics of the elementary damage flows of the model with the characteristics of the experimental flows of emission pulses and reveal features in the statistical characteristics indicating the transition of the system to the stage preceding the destruction (transition of the time autocorrelation function through a minimum in the negative range or kink on Hurst statistics).

\section{Theory}

The work of a probabilistic cellular automaton used for modelling is determined by a set of probabilities characterizing the formation of elementary damage by several complementary mechanisms and generating time series of number of elementary fractures and number of elementary damage clusters as a result of the evolution of spatial cluster structure. The probability of the formation of elementary damage on a free lattice site (occupation probability) $p_{\text {occ }}$ represents the intensity of the material destruction process under the influence of mechanical stresses averaged at spatial scales much larger than the characteristic size of the elementary fracture, and is determined by external conditions of the material loading. The probability of perimeter germination for the damage cluster $p_{s p r}$ reflects the increased intensity of the material destruction process under the action of local overstress near the already existing elementary fracture (damage cluster). The probability of merging for clusters close to critical distance $p_{\text {mer }}$ takes into account the mutual influence of a pair of damage clusters on their counter-growth. The developed cellular automaton allows realizing various scenarios of damage accumulation modelling $[2,3]$.

After selecting the simulation scenario and input parameters, each iteration of the algorithm for generating a random damage accumulation process works in the following sequence: fractures are formed on the undamaged lattice sites, the perimeters of existing clusters grow, clusters close to a critical distance merge, a cluster structure of elementary fractures is formed. Each step ends with the destruction of the cluster structure formed in the previous step and the formation of a new cluster structure, with automatic updating of all the characteristics of the clusters. The final stage of cluster structure evolution is the configuration in which a cluster that connects the opposite faces of the cube is formed (fig. 1). The formation of a connecting cluster is interpreted as the destruction of the system, and the number of iterations necessary for the formation of a connecting cluster is identified 
with the time preceding the destruction. The configuration of a cluster structure on a lattice at a specific point in time is determined by the number of clusters, as well as the characteristics of each cluster, such as mass (number of elements in a cluster), root-meansquare radius, span in rows, columns, layers. As a result, each iteration gives one point to each of the time series samples "number of elementary fractures" and "number of clusters of elementary damage", by which the characteristics of these time series are calculated.

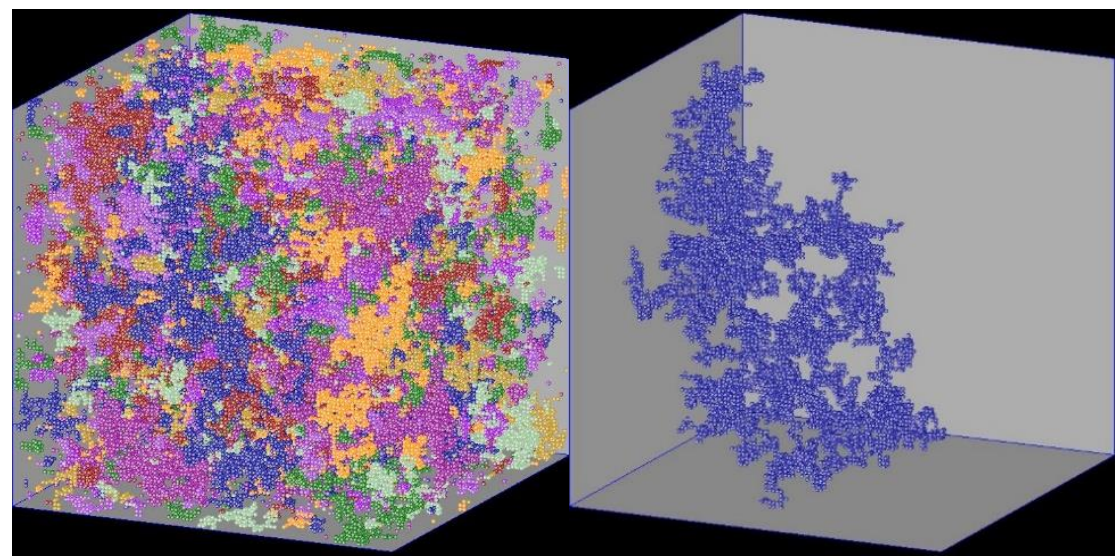

a

$\mathrm{b}$

Fig. 1. Cluster structure on the lattice $100 \times 100 \times 100$ for a dynamic internal simulation scenario before the destruction of the system (a) and the view of the connecting cluster (b).

\section{Results}

In this paper, we investigate an internal dynamic scenario that takes into account the dependence of probabilities of cluster perimeter germination on its size - the root-meansquare radius $\overline{R^{2}}$ through the stress concentration near the cluster boundary [4].

The simulation was carried out on a cubic lattice $100 \times 100 \times 100$. In this case, the probabilities had the following values: $p_{\text {occ }}=0.0001$, initial value of perimeter germination probability $p_{s p r}=0.18$, and $p_{m e r}=0.2$. The obtained characteristics of random processes were averaged over 10 realizations.

The behaviour of the time series "number of elementary fractures" essentially depends on the choice of modelling parameters. For a dynamic internal scenario, the kinetics of elementary damage accumulation in a three-dimensional model depending on the probability of damage clusters perimeter germination $p_{s p r}$ shows two qualitatively different modes of cluster structure evolution. So, for the case when the initial value of the perimeter germination probability does not exceed the value of 0.2 , the time series "number of elementary fractures", displaying an increase in the first steps of evolution, fluctuates near the mean value practically not subject to the trend. The number of damage clusters far exceeds the number of newly emerging elementary fractures (Fig. 2a). In the time series "number of elementary fractures" there are long-term correlations (Fig. 2b) with the output of the correlation function in the negative range of values. For germination probability $p_{s p r}=0.2$ the number of newly arising elementary fractures and the number of damage 
clusters coincide in order of magnitude. The formation of the connecting cluster is accelerated by about three times. The processes that form the time series "number of elementary fractures" become more coincident with each other. For the probability values of perimeter germination for clusters exceeding 0.2 , the process of combining elementary damage and the occurrence of a connecting cluster is so accelerated that the number of newly occurring elementary fractures is several times greater than the number of clusters of elementary damage (Fig. 3a), and, accordingly, the number of steps before connecting is significantly reduced. The processes that form the time series "number of elementary fractures" and the process of cluster formation become completely coincident. Correlation functions also almost coincide which indicates the occurrence of strong long-term correlations (Fig. 3b).

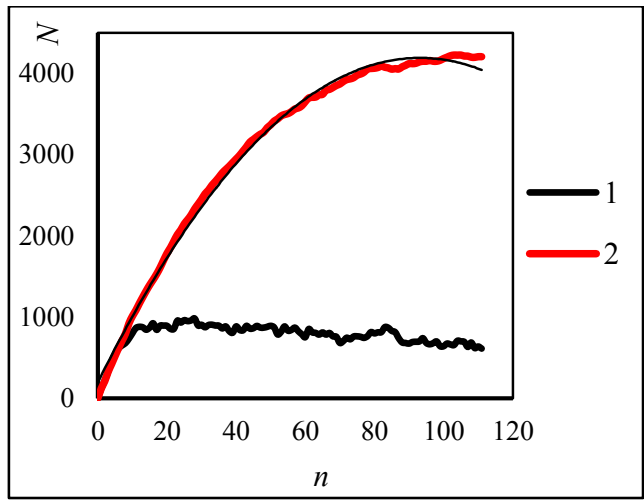

a

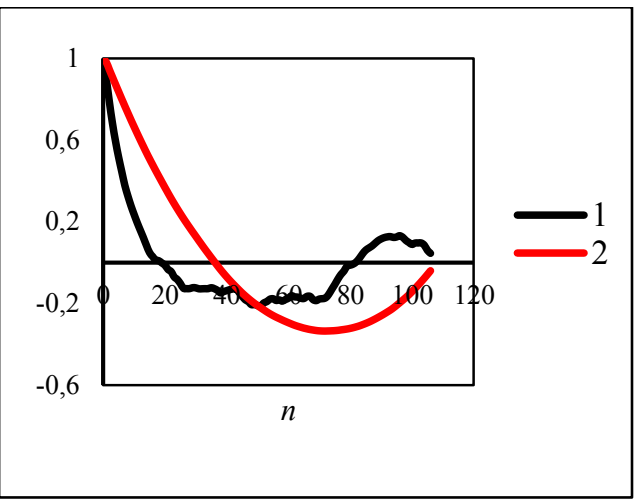

b

Fig. 2. Kinetic dependences (a) and autocorrelation functions (b) of time series: 1 - "number of elementary fractures"; 2 - "number of damage clusters" at initial value $p_{s p r}=0.18$.

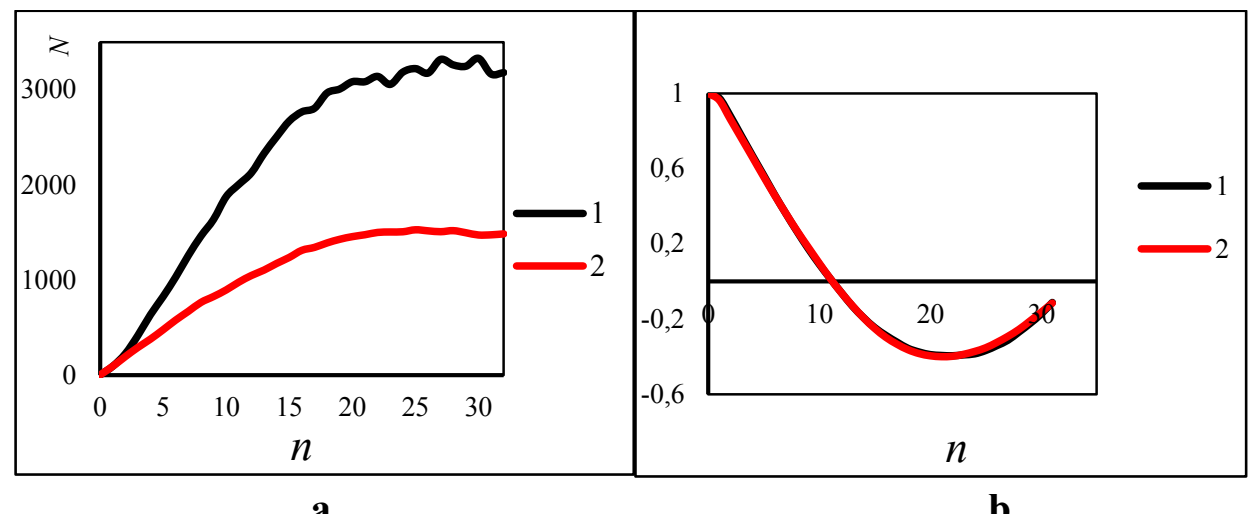

Fig. 3. Kinetic dependences (a) and autocorrelation functions (b) of time series: 1 - "number of elementary fractures"; 2 - "number of damage clusters" at initial value $p_{s p r}=0.22$.

Since the time series "number of elementary fractures" corresponds to the flow of emission pulses, which carries information about the occurrence of new and germination of previously formed microcracks, we compared the behaviour of the time series "number of elementary fractures" obtained by simulation with the characteristics of an electromagnetic emission pulse flow measured in a physical experiment. Fig. 3 compares the autocorrelation 
functions of the time series "number of elementary fractures" calculated from the data of a model experiment with the time autocorrelation functions of the electromagnetic emission pulse flows obtained during loading quartz diorite and porphyrite [5]. Autocorrelation functions were calculated by the standard formula [4].

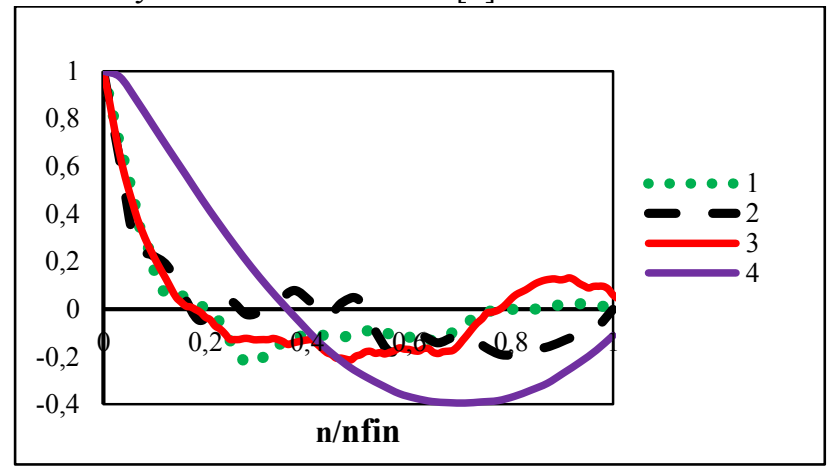

Fig. 4. Autocorrelation functions of the time series "number of elementary fractures":

1 - porphyrite, electromagnetic emission; 2 - quartz diorite, electromagnetic emission; 3 - dynamic internal script $p_{s p r}=0.18 ; 4-$ dynamic internal script $p_{s p r}=0.22$.

As it can be seen from fig. 3, the behaviour of the autocorrelation functions of the number of electromagnetic emission pulses qualitatively coincides with the behaviour of the autocorrelation function of the time series "number of elementary fractures" for the evolution mode of the cluster structure corresponding to the probability of perimeter germination smaller than 0.2 .

\section{Conclusions}

It is established that, depending on the probability value of damage cluster perimeter germination for a three-dimensional damage accumulation model, two qualitatively different modes of evolution of damage accumulation process are observed. For perimeter germination probability values exceeding 0.2 , the process of transition to irreversible destruction is significantly accelerated and becomes strongly correlated. In this case, the best correspondence between the data of model and physical experiments is observed for the mode of cluster structure evolution, corresponding to the probability of perimeter germination smaller than 0.2 . The study allows us to compare the simulation data with the data of physical experiment more reliably in order to develop methods for continuous monitoring of fracture prediction on the transition of the autocorrelation functions of pulsed emission flows to the range of negative correlation.

\section{References}

1. M. V. Kurlenya, A. P. Vostrecov, G. I. Kulakov, G. E. Yakovickaya, Registration and processing of signals of electromagnetic radiation of rocks, (Novosibirsk, 2000) $232 \mathrm{pp}$. [in Russia].

2. L. R. Botvina, Destruction: kinetics, mechanisms, General laws, (Moscow, 2008) 334 pp. [in Russia].

3. D.V.Alekseev, G.A. Kazunina, Journal of Mining Science, 42, 1 (2006). 
4. D.V. Alekseev, G. A. Kazunina, A. V. Cherednichenko, Journal of Mining Science, 51, 5 (2015).

5. G. A. Kazunina, A. A. Mal'shin, Russian Physics Journal, 52, 6 (2009). 